\title{
Analysis and Forecast of Grain Supply and Demand in China's thirteen-five Period
}

\author{
Wei-Ya SHI, Tie-jun YANG and Yi-Tao LIANG \\ College of Information Science and Engineering, Henan University of Technology, Zhengzhou 450001, China
}

\begin{abstract}
In recent years, the demand and supply of grain has dramatically changed. The paper uses the intelligent information processing method to analyse grain supply and demand of China. The gray model and Back Propagation neural network are used to forecast the trend of grain supply and demand in the thirteen-five period. The result shows that both grain supply and demand will increase in long trend, but grain demand has more than grain supply. The forecasting grain supply growth ratio will be separately $0.88 \%$ and $0.79 \%$, the grain demand will be increased $32.2 \%$ and $19.5 \%$ in 2020 using the grey model and BP, compared with the supply and demand in 2014. At last, the suggestions are given. In order to guarantee the grain safety, scientific method should be used to monitor and analysis the grain market.
\end{abstract}

\section{Introduction}

The grain is the main portion of people daily lives. The food security is the foundation for national stability. It is especially important for china who has about 1.4 billion population, but only has seven percent of world arable land.

The demand and supply of grain has dramatically changed at different times. Many scholars at home and abroad had done many qualitative and quantitative researches. Wang [1] had proposed to give responsible forecast in advance of food production by analyzing grain yields from 1978 to 2004 in China. Xie [2] had found that grain yield is closely related to the cultivating acreage, usage of chemical fertilizer, political factor using linear regression model. Sun [3] had built forecasting system to give prediction for grain demand and pointed out there had gap between demand and supply in future. Zhao [4] had used GARCH and ARMA model to forecast the future trend of grain and found the shortage will last for many years. Gu [5] also investigated many influential factors to analyze the demand and supply situation. There are also some researchers [6,7] who considered the relation between grain production and many other factors, such as climate, water resource. In Wu's dissertation, the $\mathrm{GM}(1,1)$ model are constructed to simulate the medium and long term trend of grain demand and supply. Zhang [9] used different grain variety to forecast the demand from 2007 to 2030 and found that the grain demand will have sustainable growth in China.

The problem of grain demand and supply has increasingly serious with the expansion of city scale, the increase of extreme weather, the growth of population and many other uncertainties. How to efficiently solve the imbalance between supply and demand of grain is directly related to national food security. However, it cannot adapt to the new conditions only using the traditional historical experience and some simple statistical computation. This paper uses the intelligent information processing method to investigate the problem of supply and demand of grain and gives the forecasting model. The model can provide service for government to adjust the grain market.

\section{The History and Present Situation Analysis of China Grain Supply and Demand}

Considering the historical situation, natural disaster and the Cultural Revolution in China, the paper only uses the data since 1978 .

Figure 1(a) gives the trend of grain yield from 1978 to 2013. It can be found that the grain yield has a growth trend in general. Figure 1(b) is the clustering result using K-mean algorithm, which shows four stages denoted by '*', ', ', $\diamond$ 'and ' $\square$ '. 


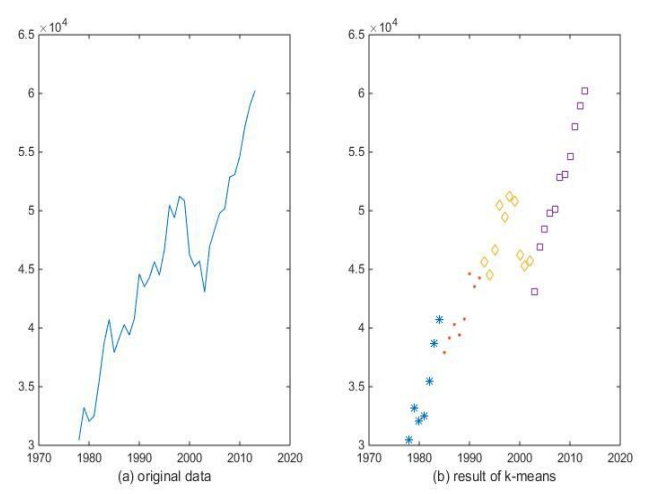

Figure 1. Historical trend of grain yield and clustering result using k-means

The description of four stages is given as follows:

- First stage: The grain yield has quickly increased from 1978 to 1984 . The main reason is that grain yield per unit area has a fast growth. According to national statistical yearbook, although the national grain sown area has declined $6.4 \%$, grain yield per unit area in 1984 increased about $42.8 \%$ compared to one in 1978.

- Second stage: The grain yield has also similarly increased from 1985 to 1992 . But the growth rate has slightly declined. The main reason also comes from the grain yield per unit area.

- Third stage: The grain yield has declined from 1993 to 2003. It dues to that the national grain sown area has reduced in a large extend.

- Fourth stage: The grain yield has quickly increased from 2003 to 2013. The growth of grain sown area and grain yield per unit area can explain the fact.

Table 1 gives the mean of different stage.

Table 1. Clustering result of grain yield using K-means

\begin{tabular}{|l|l|}
\hline $\begin{array}{l}\text { period of time } \\
\text { (year) }\end{array}$ & $\begin{array}{l}\text { Average grain yield } \\
\text { (Ten thousand tons) }\end{array}$ \\
\hline $1978--1984$ & 34736 \\
\hline $1985 \sim 1992$ & 41423 \\
\hline $1993--2002$ & 47595 \\
\hline $2003--2013$ & 52296 \\
\hline
\end{tabular}

The national grain demand generally consists of food consumption, feed consumption, seed consumption, industrial consumption and grain loss. With the improvement of people's living standard, the food consumption has been declining gradually in recent years. But the industrial consumption and feed consumption has a faster growth. For example, the food consumption, feed consumption and industrial consumption was respectively 25389, 14722 and 10130 ten thousand tons in 2012, which has a comparable growth of $6.9 \%, 23.1 \%$ and $123.8 \%$ in 2003 .

\section{Forecast of Grain Demand and Supply}

The paper respectively uses back propagation neural network [11-13] and grey model [14-16] to give predication of grain demand and supply in China's thirteenth five-year development planning.

\subsection{Basic Concepts of BP}

BP (Back Propagation) neural network is one of neural network model which has been extensively used. It uses the algorithm of gradient descent and adjusts the weight value based on back propagation. Its goal is to find a mapping between inputs and its correct output. Figure 2 gives the structure of neural network, which is generally composed with input layer, hidden layer and output layer. Input layer is in charge of receiving the external information, hidden layer is responsible for the information processing, and the output layer gives the finally result.

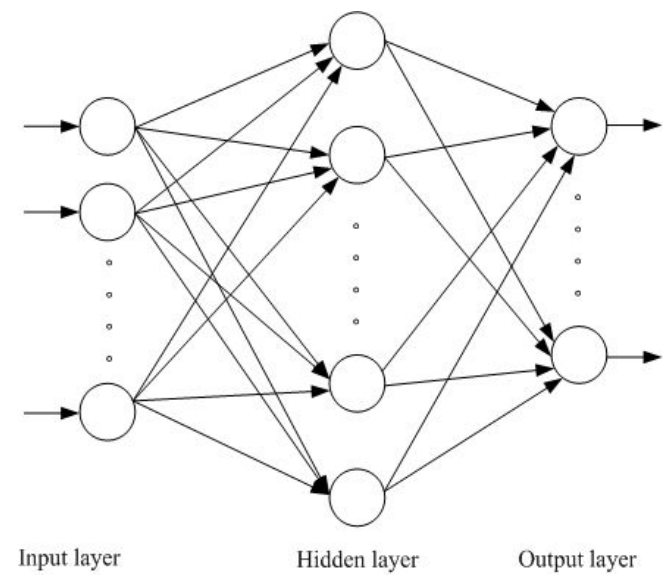

Figure 2. Structure of Neural Network

The BP algorithm can be outlined into two parts: forward and back propagation. In the part of forward propagation, the input information is transferred to output layer and given the output. When the output is inconsistent with correct result, error propagates to the forward neurons. In the whole learning process, the weights and bias values are continuously updated until error is less than predefined value. The learning parameters are weights and bias values.

\subsection{Basic Concepts of Gray Model}

The gray model is firstly proposed in 1982. After that, the gray theory has been rapidly developed and caught the attention of many scholars. It has been widely used in various fields.

In the gray theory, a system can be defined according to its color, which represents the different degree of information about that system. For example, a system is called white system if all information about it has been known. Similarly, if we has any idea about one system, we denoted it black system. If part information has been known, the system is the grey system. In real application, because each system has some uncertainties, it can be regarded as a grey system.

The gray forecasting model has many advantages, for example, it needs less information about the system, in addition, the gray model has the characteristic of 
convenient computation and high accuracy. The main goal of gray model is to extract realistic principles of the system using available data.

\subsection{The Dataset}

Selection of sample data: the sample data mainly comes from China Statistical Yearbook, State Statistics Bureau, and website of United Nations Food and Agriculture Organization (FAO). The grain yield data is selected from 1978 to 2013. We only consider the food consumer price index from 1995 to 2013 for the sake of statistical cause.

\subsection{Trend Forecast of Grain Supply}

In the experiment, the grey model uses all historical data to forecast next year grain yield. Autoregressive order is 10 in the neural model. The neuron of hidden layer is 10 . The tan-sigmoid and purelin function are separately chosen as the transferring function for hidden and output layer of neural network. Seventy percent of raw data are used as training sample and the others are used as testing samples.

Table 2. Forecasting Result of Grain Yield Using Grey Model And $\mathrm{Bp}\left({ }^{*} \mathrm{E}+04\right)$.

\begin{tabular}{|l|l|l|}
\hline year & Grey model & BP \\
\hline 2014 & 5.8086 & 6.3402 \\
\hline 2015 & 5.8910 & 6.6468 \\
\hline 2016 & 5.9745 & 6.7205 \\
\hline 2017 & 6.0592 & 6.7621 \\
\hline 2018 & 6.1451 & 6.8319 \\
\hline 2019 & 6.2322 & 6.8458 \\
\hline 2020 & 6.3206 & 6.8473 \\
\hline
\end{tabular}

Table 2 and figure 3 give the result. The error of grey model and BP neural network is $5.22 \%$ and $4.96 \%$, respectively. From the result, both of two methods predict that the grain yield will increase in short term. The forecast growth ratio will be separately $0.88 \%$ and $0.79 \%$ in 2020 using the grey model and BP, compared with the yield in 2014. But the BP give more smooth result than the grey model.
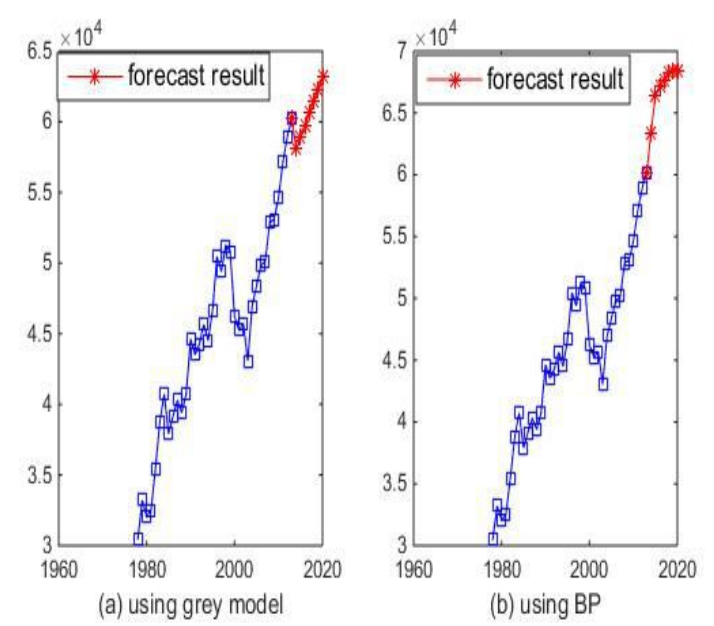

Figure 3. Forecasting Result of Grain Yield Using Grey Model and $\mathrm{Bp}$

\subsection{Trend Forecast of Grain Demand}

Because there has not the exact statistical data for grain demand, the food consumer price index is considered as index to judge grain demand.

The similar parameters are used as the experiment of grain supply. Table 3 and figure 4 give the final result. The error of grey model and BP neural network is $4.80 \%$ and $4.60 \%$, respectively. The grain demand in 2020 will be increased $32.2 \%$ in 2020 that 2014 using the grey model. But the BP neural network gives a growth of $19.5 \%$. In total, the two methods give similar result. From the view of long term, the food consumer price index will experience a sustainable growth.

Table 3. Forecasting result of food consumer price index using grey model and BP.

\begin{tabular}{|l|l|l|}
\hline year & Grey model & BP \\
\hline 2014 & 173.5 & 183.4 \\
\hline 2015 & 181.8 & 195.1 \\
\hline 2016 & 190.5 & 204.6 \\
\hline 2017 & 199.5 & 206.4 \\
\hline 2018 & 209.0 & 205.1 \\
\hline 2019 & 218.9 & 213.1 \\
\hline 2020 & 229.4 & 219.2 \\
\hline
\end{tabular}
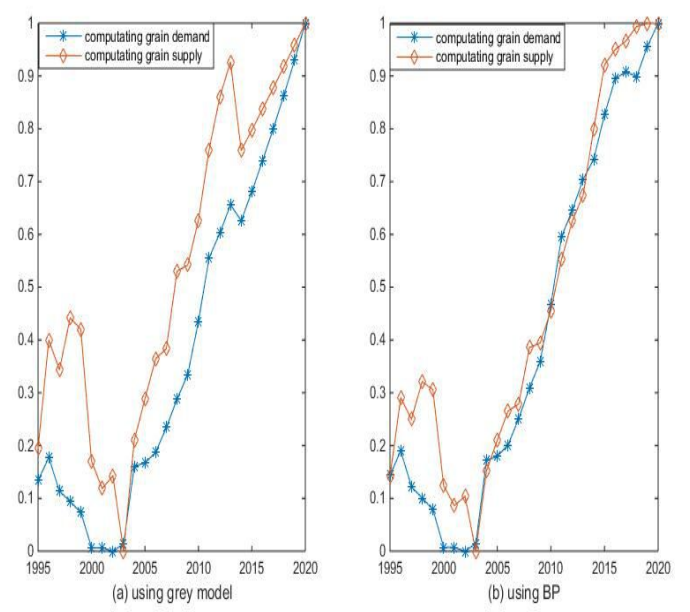

Figure 4. Forecasting Result of Food Consumer Price Index Using Grey Model and Bp 
Figure 4 also gives the forecast result for grain yield. It can be found that the grain demand has larger growth than the grain yield. It shows the demand is greater than supply and the grain supply may be tension in China's thirteenth five-year.

\section{Proposal for grain supply and demand}

In this paper, we uses gray model and Back Propagation neural network to analyses and predict grain supply and demand of China in the thirteen-five period. The result shows that both grain supply and demand will increase in long trend, but grain demand has more than grain supply.

As mentioned above, there exists the imbalance between grain supply and demand. As a result, some methods should be adopted to guarantee the balance between grain supply and demand. It has been point out that government should build grain early warning and monitoring system and establish a sound and efficient grain control mechanism in the Long-term plan for China's food security (2008-2020).

In order to maintain the balance of grain supply and demand, the following methods can be implemented.

technology and steadily improve the comprehensive grain production capacity.

and build sound grain macro control system.

system and establish early warning model and index system of grain supply and demand balance.

policy to protect and enhance the enthusiasm of farmers.

reduce food waste.

though International grain market. Promote grain trade balance and macroeconomic regulation and control of grain prices

\section{Acknowledgements}

This work was supported in part by Special Fund for Agro-scientific Research in the Public Interest under contract 201413001, the National Natural Science Foundation of China under contract 31171775, Natural Science Foundation of Henan Educational Committee under contract 14B550002, and the key technologies R\&D program of Henan science and technology committee under contract 112102210190.
2. Jie Xie, Study on the influencing factors of grain production in China, inquiry into economic issues, 36-40,9(2007)

3. Baomin Sun, Prediction index system and model design of grain supply and demand in China, economic issue, 39-43,3(2012)

4. Xuan Zhao, Yishan Shao, Analysis and prediction of grain supply and demand in China, Research of Agricultural Modernization, 277---280,3(2014)

5. Guoda Gu, Jinghua Yi, Analysis on the trend of global medium and long term grain supply and demand, Journal of Huazhong Agricultural University (Social Science Edition), 6-16,6(2014)

6. HUEY L L, The impact of climate change on global food supply and demand, food prices, and land use, Paddy Water Environ, 321-331,7(2009)

7. HANJRA M A, QURESHIM E, Global water crisis and future food security in an era of climate change, Food Policy, 365-377,10(2010)

8. Le $\mathrm{Wu}$, Research on the long term trend of China's grain demand, Doctoral Dissertation of Huazhong Agricultural University, (2011)

9. Yumei Zhang, Yuqiang Li, Zhemin Li, Shiwei Xu, China Grains Demand Forecast Analysis Based on China Economywide Multimarket Model, Food and Nutrition in China, 40-45,2(2012)

10. Bishop, Christopher M, Pattern Recognition and Machine Learning, Springer (2010)

11. Simon Haykin, Neural Networks and Learning Machines, China Machine Press, (2011)

12. Alpaydın, Ethem, Introduction to machine learning (2nd ed. ed.), Cambridge, Mass.: MIT Press, 2010.

13. Rumelhart, David E., Hinton, Geoffrey E., Williams, Ronald J, Learning representations by backpropagating errors, Nature. 323 (6088) (1986) 533536.

14. Julong Deng, Grey theory foundation, Huazhong University of Science and Technology Press, (2005)

15. Xiu Wang, Comparative study on grain yield prediction of grey forecasting model, journal of agricultural mechanization research, 78-80,9(2011)

16. Erdal Kayacan, Baris Ulutas, Okyay Kaynak, Grey system theory-based models in time series prediction, Expert Systems with Applications, 17841789,37(2010)

\section{References}

